\title{
Microstrip Ultra-Wideband Filter with Flexible Notch Characteristics
}

\author{
Marjan Mokhtaari, Jens Bornemann
}

Department of Electrical and Computer Engineering, University of Victoria, Victoria, Canada.

Email: j.bornemann@ieee.org

Received October $1^{\text {st }}, 2011$; revised November $2^{\text {nd }}, 2011$; accepted November $10^{\text {th }}, 2011$

\begin{abstract}
A microstrip ultra-wideband (UWB) filter with unique shape, compactness, simplicity of operation and flexible notch characteristics is introduced. It is based on the fundamental and harmonic characteristics of a $50 \mathrm{Ohm}$ transmission line that is grounded at both ends. The filter possesses design flexibility in the sense that it can operate as a stand-alone UWB component or include simple additional circuitry to create one or two notches within the ultra-wideband frequency range. The basic design principles are highlighted and verified using the results of two commercially available field solver packages. Individual filter structures with single and double notches are validated through measurements of a number of filter prototypes.
\end{abstract}

Keywords: Microstrip Filters; Microstrip Resonators; Bandstop Filters; Ultra-Wideband Filters

\section{Introduction}

Ultra-wideband (UWB) applications attract increasing attention, both in industry and academia, due to increasing levels of sophistication and demand for advanced communication systems, e.g. [1]. One of the key issues in the $3.1-10.6 \mathrm{GHz}$ range is the interference from wireless local area networks (WLANs) between 5 $\mathrm{GHz}$ and $6 \mathrm{GHz}$. Therefore, general UWB filters, but especially those incorporating notch capabilities, are in demand [2]. Several conventional UWB filter design approaches have been introduced, e.g. [3-5]. The introduction of tunable harmonic stepped-impedance resonators (SIWs) initiated a new generation of UWB filter designs, e.g. [6]. The common critical issue in these approaches, however, is their high manufacturing accuracy due to tightly coupled segments, as they are required to perform over the entire bandwidth [7,8]. Other designs focus on the utilization of defected-ground planes to enhance UWB band-stop specifications, e.g. [9], or SIWs with shortcircuit stubs for dual-band applications [10].

In order to eliminate interference from other services within the UWB band, a UWB filter must provide additional narrowband rejection capability in the passband. One solution to meet this specification is to utilize conventional open-ended quarter-wavelength transmission lines, which reject signals at that specific frequency $[11,12]$. A number of additional options, including those involving technologies other than microstrip, are dis- cussed in [2].

This paper introduces a compact UWB microstrip filter, which is not only easy to prototype but also provides design flexibility for single and double notching within the UWB passband. The stand-alone UWB filter follows from work recently presented in $[13,14]$ where also tuning capability with respect to a certain notch configuration is demonstrated. The current paper presents new possibilities of creating single or double notches while maintaining the circuit dimensions of the original UWB filter. Several prototype measurements validate the design approach and the additional circuitry for notch creation.

\section{Design Guidelines}

\subsection{Stand-Alone UWB Filter}

Figure 1(a) shows the layout of a triple-resonator microstrip filter whose operation is based on the resonance characteristic of a $50 \mathrm{Ohm}$ transmission line which is grounded by via holes at both ends [13]. Its basic operation is explained as follows (Figure 1(b)). Length $\mathrm{L}$ is chosen to be a half wavelength at center frequency of $\mathrm{f}_{0} \approx$ $6 \mathrm{GHz}$. Lengths $\mathrm{L}_{1}$ are quarter-wavelengths sections at the same frequency so that the entire length forms a fullwavelength via-grounded transmission line. The impedance of the main transmission line $\mathrm{L}$ and input/output sections is $50 \mathrm{Ohm}$. Source (input) and load (output) are tapped to the resonator at points where all three modes 


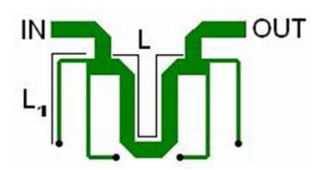

(a)

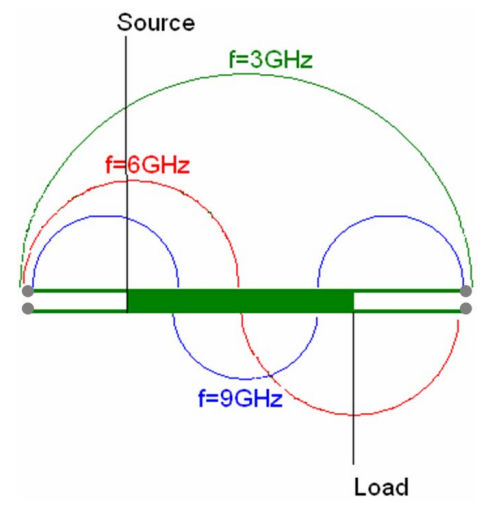

(b)

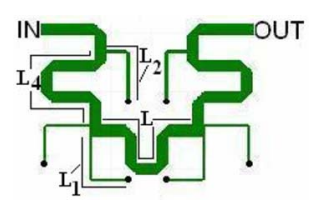

(c)

Figure 1. (a) Layout of triple-resonator microstrip filter; (b) Explanation of fundamental resonance and first and second harmonics at around $3 \mathrm{GHz}, 6 \mathrm{GHz}$ and $9 \mathrm{GHz}$, respectively; (c) Layout for bandwidth enhancement.

are excited (Figure 1(b)). The quarter-wavelength sections $L_{1}$ are realized as two parallel segments of 100 Ohms each. This is not a necessary condition for the operation of the triple-mode resonator but has shown some minor benefit in the design process as it provides slightly increased flexibility. Ideally, these segments have an impedance of $100 \mathrm{Ohms}$ and support a compact size through structural folding. For ease of fabrication, though, their minimum width might be limited. Therefore, their minimum width is set to, e.g., $100 \mu \mathrm{m}$, thus resulting in slightly lower impedance. Alternatively and depending on the substrate used, impedances are limited to 100Ohm lines if they lead to line width $\leq 100 \mu \mathrm{m}$.

In addition to the filter of Figure 1(a), Figure 1(c) depicts sections $\mathrm{L}_{2}$ and $50 \mathrm{Ohm}$ lines $\mathrm{L}_{4}$ in order to increase the bandwidth of the UWB filter. The sections of length $\mathrm{L}_{4}$ operate as quarter-wavelength impedance inverters at $3 \mathrm{GHz}$ and as half-wavelength resonators at $6 \mathrm{GHz}$. Lengths $L_{2}$ are a quarter-wavelength long at around 6 $\mathrm{GHz}$. Their impedance level and/or width are selected in the same way as sections $\mathrm{L}_{1}$. Using RT/Duroid 6010 substrate with $\varepsilon_{\mathrm{r}}=10.2$, substrate height $\mathrm{h}=635 \mu \mathrm{m}$ and metallization thickness $\mathrm{t}=35 \mu \mathrm{m}$, the overall dimensions of the filter structure in Figure 1(c) are $11 \mathrm{~mm} \times 8 \mathrm{~mm}$.

Figure 2(a) shows the responses of the filter in Figure

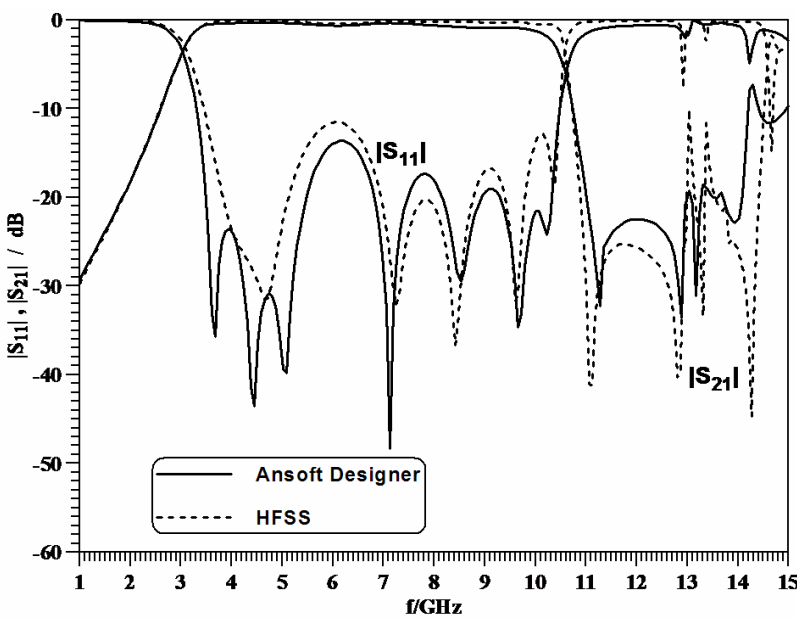

(a)

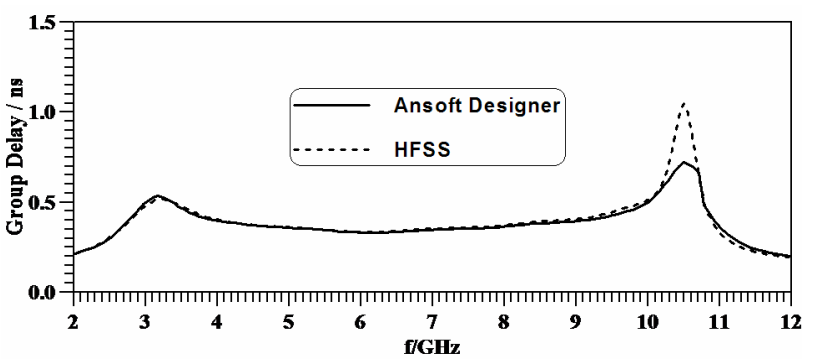

(b)

Figure 2. Scattering parameters (a) and group delay (b) of the UWB microstrip filter in Figure 1(c); performance comparison between Ansoft Designer and HFSS.

1(c) obtained with Ansoft Designer and the High-Frequency Stucture Solver (HFSS). (Ansoft Designer and HFSS are two different commercially available field solvers, the first based on the method of moments, the latter on the finite element technique. They are used together in this paper for the purpose of result validation as a single numerical technique, depending on its specific implementation, is often not an indication of a reliable design.) Good agreement is obtained for the passband and most of the stopband. According to Ansoft Designer, the 20dB band-stop region extends from $11 \mathrm{GHz}$ to $14.15 \mathrm{GHz}$. The transmission zero at $11.3 \mathrm{GHz}$ is due to the fact that the input/output placements along the grounded transmission-line resonator create an out-of-phase feeding scenario similar to that discussed in [15]. (Note that his transmission zero is inherent in the design and appears in all filter responses shown in this paper.) The $3 \mathrm{~dB}$ bandwidth extends from $3 \mathrm{GHz}$ to $10.4 \mathrm{GHz}$ and covers almost the entire UWB frequency range. Figure 2(b) shows the group delay response of the filter in Figure 1(c). In the passband, it is derived from the computed or measured transmission phase $\varphi_{21}$ as

$$
\tau=-\partial \varphi_{21} / \partial \omega
$$


In the stopband or at transmission zeros, the output signal is diminished due to high attenuation, and thus the group delay is extracted using the phase $\varphi_{11}$ of the reflection coefficient and the properties of lossless symmetrical two-port devices.

$$
\varphi_{11}=\varphi_{21} \pm \pi / 2
$$

Since the group delay is meaningful only at passband frequencies, Figure 2(b) (and following group delay plots) focuses mainly on the ultra-wide passband. For the circuit in Figure 1(c), good agreement in and close to the passband is observed in Figure 2(b). The group delay variation is less than 200 ps as confirmed by Ansoft Designer and HFSS and is thus better than or comparable with many UWB filters presented in the recent literature.

\subsection{UWB Filter with Notch Capability Specifications}

Up to this point, we were concerned with the filter as a stand-alone UWB component. Now the creation of a notch in the frequency response is demonstrated. This is achieved by adding open-ended coupled-line sections $\mathrm{L}_{3}$ and tapping them off lines $\mathrm{L}_{2}$ at a distance $\mathrm{L}_{5}$. A comparison between Figures 1(c) and 3(a) illustrates the concept. The open and shorted stubs formed by lengths $\mathrm{L}_{2}$ in Figure 1(c) and $\mathrm{L}_{3}, \mathrm{~L}_{5}$ in Figure 3(a) have band-stop characteristic and thus create the notch in the frequency response. Note that the notch is absent in Figure 2(a) because lines $\mathrm{L}_{2}$ in Figure 1(c) are too short for the UWB frequency range. For design purposes, length $\mathrm{L}_{3}$ in Figure 3(a) is determined as

$$
L_{3}=\left(\lambda^{\prime} / 4\right)-L_{5}
$$

where $\lambda^{\prime}$ is the guided wavelength at the desired notch frequency. Figure 3(b) and (c) show the scattering and group delay performances, respectively, for this filter. The notched $3 \mathrm{~dB}$ bandwidth in Figure 3(b) is $800 \mathrm{MHz}$, covering the frequency range from $5.2 \mathrm{GHz}$ to $6 \mathrm{GHz}$, and the maximum attenuation is $30 \mathrm{~dB}$. Other than that, the performance of the filter in Figure 3(b) is similar to that in Figure 2(b). The group delay performance in Figure 3(c) shows variations of less than 100 ps and 200 ps for the first and second passbands, respectively.

Tuning of the notch is facilitated by varying lengths $\mathrm{L}_{3}$, $\mathrm{L}_{5}$ and adjusting the spacing "s" between the coupled lines. The $3 \mathrm{~dB}$ bandwidth of the notch is adjusted mostly by $L_{5}$, but also slightly by $L_{3}$, which then changes in the opposite direction according to Equation (3). In addition, the reflection coefficients within the two passbands (initial UWB filter response now separated by the notch) are slightly adjusted by the gap "s" between the open-ended coupled segments of length $\mathrm{L}_{3}$. This has been initially

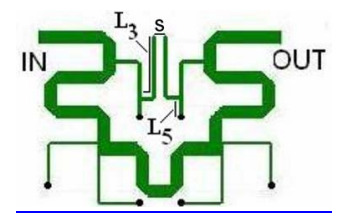

(a)

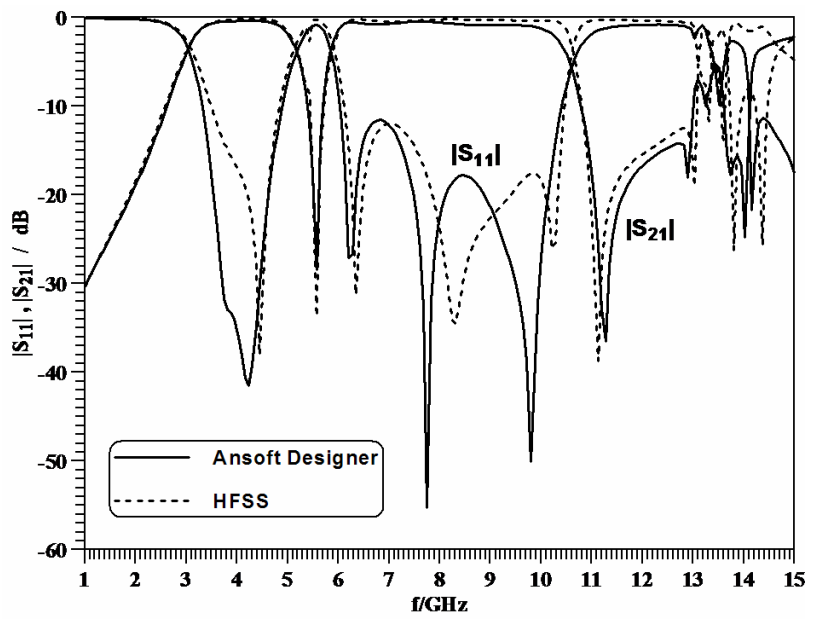

(b)

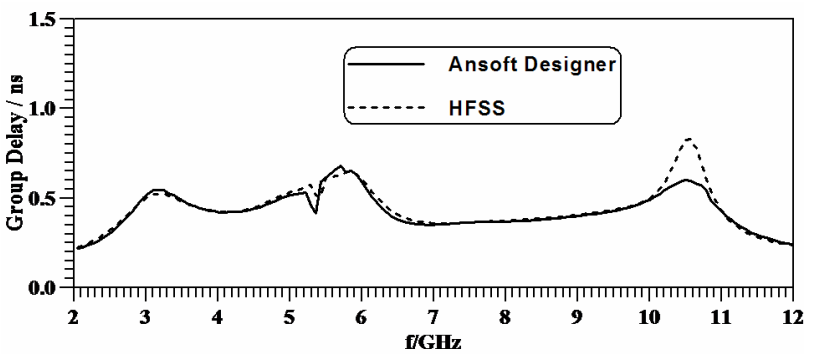

(c)

Figure 3. Layout (a), scattering parameters (b) and group delay (c) of the UWB microstrip filter with notch characteristic; performance comparison between Ansoft Designer and HFSS.

demonstrated in a parametric study presented in [14].

\section{Results}

This section shows some of the results obtained with selected prototypes. About 16 different designs were implemented on a single RT/Duroid 6010 substrate with $\varepsilon_{\mathrm{r}}$ $=10.2$, substrate height $\mathrm{h}=635 \mu \mathrm{m}$ and metallization thickness $\mathrm{t}=35 \mu \mathrm{m}$. They were cut to individual filter units, and coaxial connectors were soldered to input and output ports as a test fixture was not available. One of the problems in this prototyping approach is the fact that the input and output coaxial adapters, as shown in Figure 4 (a), are located very close to the actual filter circuit. They are responsible for the high level of reflection seen in the passbands in Figure 4(b) and the somewhat wavy group delay response in Figure 4(c). (Similar behavior is ob- 


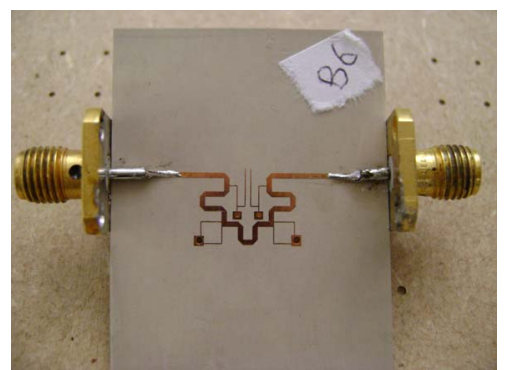

(a)

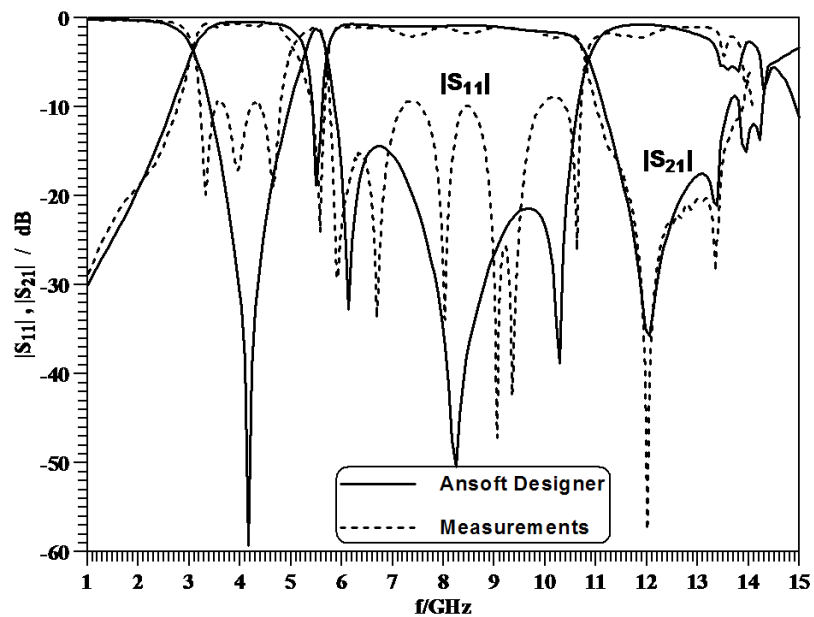

(b)

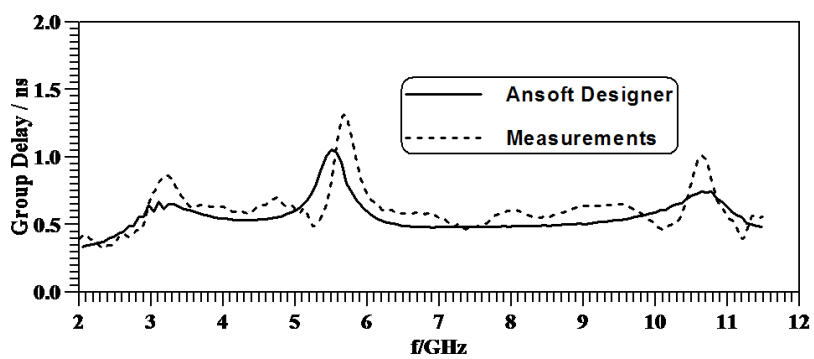

(c)

Figure 4. Photograph (a), measured $S$ parameters (b) and group delay (c) of a UWB microstrip filter with notch characteristic; performance comparison with Ansoft Designer.

served for all following measurements in this section.) Other than that, the agreement between computation and measurements is very good. The measured passband return loss is about $10 \mathrm{~dB}$, and the notch frequency at 5.55 $\mathrm{GHz}$ and the transmission zero at $12 \mathrm{GHz}$ are well represented in the experiment.

According to Figure 3(a) and the discussion in the previous section, a notch in the UWB filter performance can be created by employing open and shorted stubs between the input and output paths and designing their lengths and position for the notch frequency. However, the position and character of the stubs is not limited to that shown in Figures 3(a) and 4(a). For instance, Figure 5(a) shows coupling directly across the original

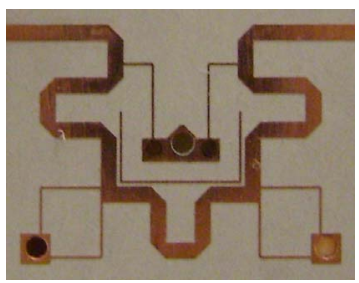

(a)

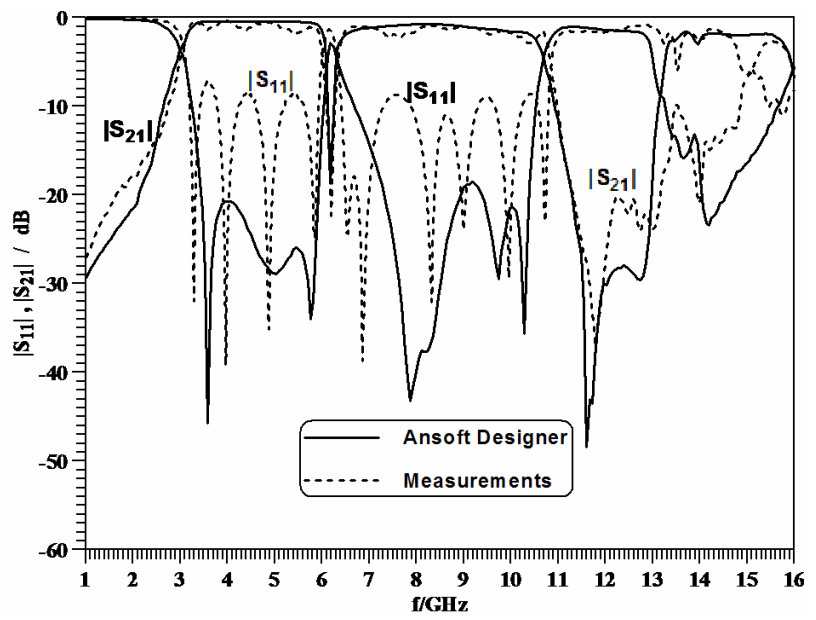

(b)

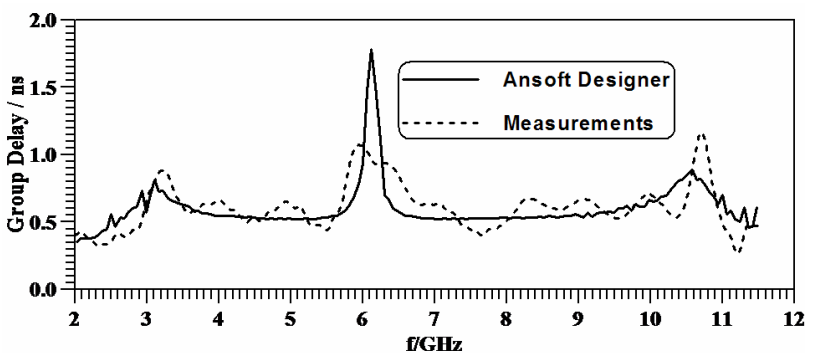

(c)

Figure 5. Photograph (a), measured $S$ parameters (b) and group delay (c) of a UWB microstrip filter with notch characteristic using an alternative coupling scheme; performance comparison with Ansoft Designer.

triple resonator (Figure 1(a)) whereas the upper filter part is similar to Figure 1(c) with the exception that the two vias at the end of lengths $\mathrm{L}_{3}$ are realized by a combination of vias. The length of the coupling path is a halfwavelength at the notch frequency. The measurements in Figures 5(b) and (c) show similar characteristics as the previous ones. The measured return loss is about $8 \mathrm{~dB}$, and the notch at $6.2 \mathrm{GHz}$ and the overall transmission characteristics are well reproduced in the experimental data.

Double notches in the UWB filter response can be created by using the circuit in Figure 4(a) and adding another half-wave resonator between input and output. This is demonstrated in Figure 6(a). The former thus produces the notch at $5.45 \mathrm{GHz}$ while the latter adds that 


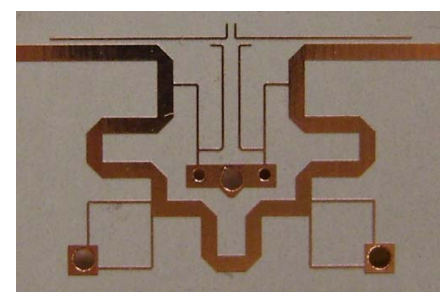

(a)

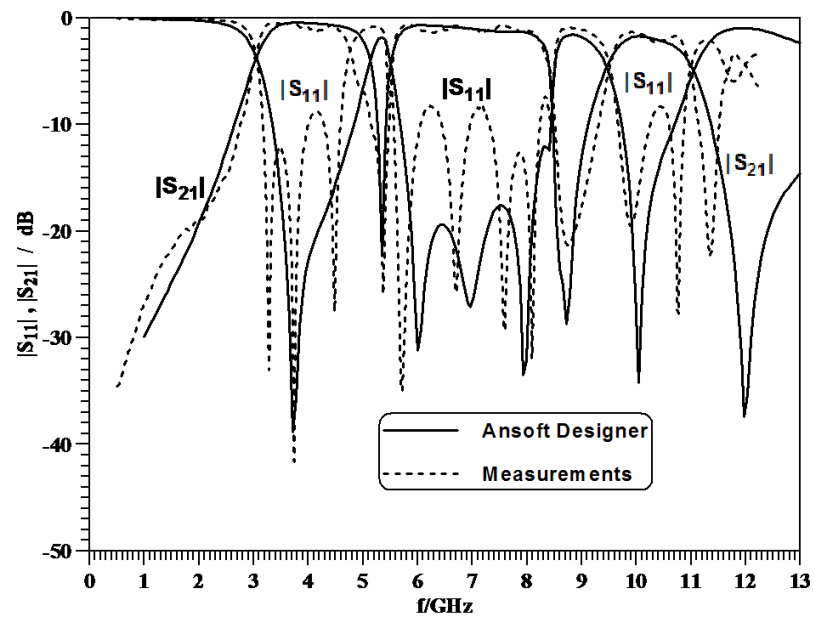

(b)

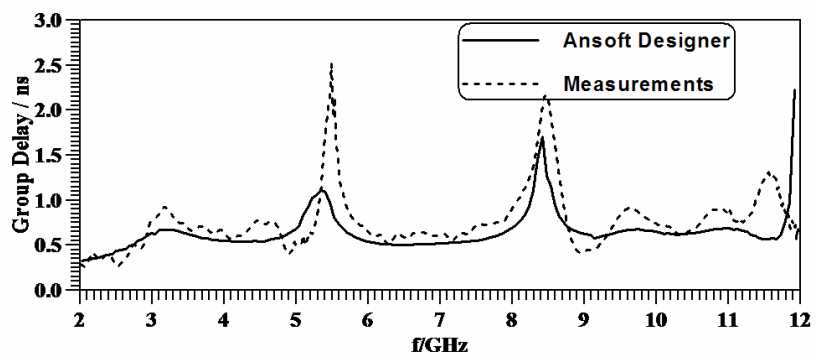

(c)

Figure 6. Photograph (a), measured S parameters (b) and group delay (c) of a UWB microstrip filter with double notch characteristic; performance comparison with Ansoft Designer.

at $8.75 \mathrm{GHz}$. Although the upper notch band is wider relative to the notch center, the UWB response is clearly divided into three separate passbands as indicated by " $\left|\mathrm{S}_{11}\right|$ " in Figure 6(b), and the measured filter transmission characteristic is in good agreement with predictions. The group delay performance in Figure 6(c) also reflects the triple-band filter characteristic.

Similar to the single-notch designs, the coupling schemes for the double notch can now be changed. Note that the dimensions of the basic $50 \mathrm{Ohm}$ resonator line as well as those of the input/output lines are the same in all prototypes presented in this paper.

A variant of the circuit in Figure 6(a) is shown in Figure 7(a). Comparing the responses of Figures 6(b) and 7(b), it is seen that the same scheme is used to pro-

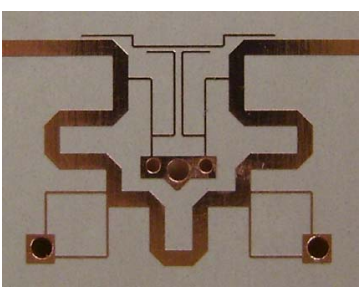

(a)

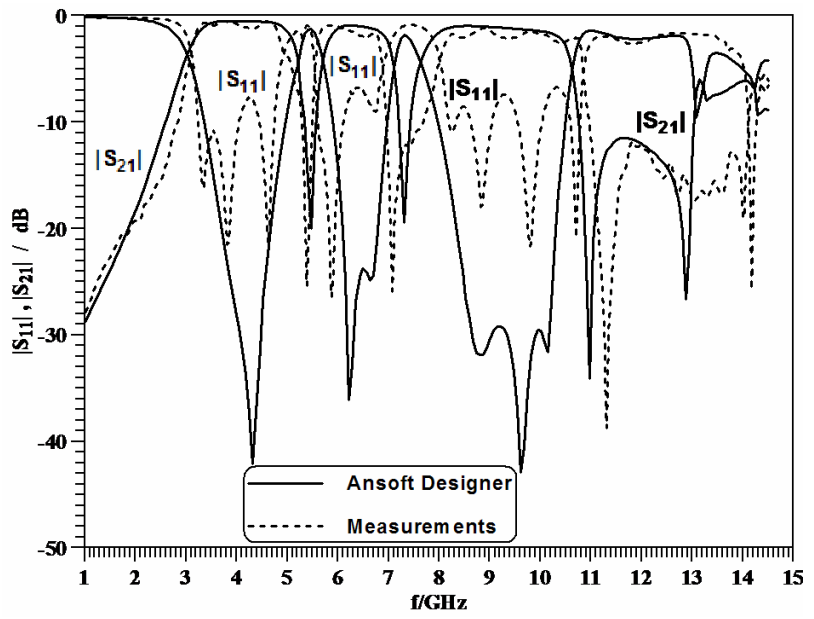

(b)

Figure 7. Photograph (a), measured S parameters (b) of a UWB microstrip filter with double notch characteristic and alternative coupling scheme; performance comparison with Ansoft Designer.

duce the lower notch at $5.45 \mathrm{GHz}$ (also c.f. Figure 4). The scheme for the upper one is altered and produces a notch designed for 7.3 GHz (Figure 7(b)). However, note that there is an uninterrupted line between the input and output coupling sections and that, compared to Figure 6(a), the coupling of the lower-notch lines to the upper one is stronger in Figure 7(a). It is believed that this is the reason for this configuration being more sensitive to tolerances. This fact explains the shift from 7.3 $\mathrm{GHz}$ in the computation to $7.05 \mathrm{GHz}$ in the experiment (Figure 7(b)). Other than that, the notches and overall transmission characteristic are well represented and, considering the reflection effect between the coaxial adapters (as mentioned earlier), the agreement between computation and experiment is generally good.

\section{Conclusion}

A microstrip ultra-wideband filter is introduced. Due to its unique shape and compactness, it offers possibilities for single and double notch operation. The filter's centerpiece is a $50 \mathrm{Ohm}$ transmission line, grounded at both ends, plus additional $50 \mathrm{Ohm}$ and (ideally) $100 \mathrm{Ohm}$ line sections. Open and shorted lines between the input and output halves of the filter facilitate a narrow notch band. 
Additional coupled half-wave resonators generate additional notches at different frequencies. The applications of the circuits are two-fold: They can be used for interference cancelation of other services in the UWB frequency range, or they can operate as dual-or triple-band filters within the $3-10 \mathrm{GHz}$ regime. Principle design guidelines determine the initial UWB filter and notch dimensions. Fine optimization with a field solver such as Ansoft Designer or HFSS is encouraged. Several prototypes are presented whose measurements, aside from the inappropriate prototyping, validate the basic filter design and the creation of notches in the frequency response.

\section{Acknowledgements}

The authors wish to acknowledge support for this work from the TELUS Research Grant in Wireless Communications.

\section{REFERENCES}

[1] J. Wells, "MM-Waves in the Living Room: The Future of Wireless High Definition Multimedia," Microwave Journal, Vol. 62, No. 8, 2009, pp. 72-84.

[2] J.-S. Hong, "Reconfigurable Planar Filters," IEEE Microwave Magazine, Vol. 10, No. 6, 2009, pp. 73-83. doi:10.1109/MMM.2009.933590

[3] G. L. Matthaei, L. Young and E. M. T. Jones, "Microwave Filters, Impedance Matching Networks and Coupling Structures," McGraw-Hill, New York, 1964.

[4] H. Ishida and K. Araki, "Design and Analysis of UWB Band Pass Filter with Ring Filter," IEEE MTT-S International Microwave Symposium Digest, Fort Worth, 6-11 June 2004, pp. 1307-1310.

[5] R. Gómez-García and J. I. Alonso, "Systematic Method for the Exact Synthesis of Ultra-Wideband Filtering Responses Using High-Pass and Low-Pass Sections," IEEE Transactions on Microwave Theory and Techniques, Vol. 54, No. 10, 2006, pp. 3751-3764. doi:10.1109/TMTT.2006.882883

[6] M. Mokhtaari, J. Bornemann and S. Amari, "A Modified Design Approach for Compact Ultra-Wideband Micro- strip Filters," International Journal of RF \& Microwave CAE, Vol. 20, No. 1, 2010, pp. 66-75.

[7] H. Wang and L. Zhu, "Aperture-Backed Microstrip Line Multiple-Mode Resonator for Design of a Novel UWB Bandpass Filter," Asia-Pacific Microwave Conference Proceedings, Vol. 1, Suzhou, 4-7 December 2005, pp. 14.

[8] M. Mokhtaari, J. Bornemann, and S. Amari, "Folded Compact Ultra-Wideband Stepped-Impedance Resonator Filters, IEEE MTT-S International Microwave Symposium Digest, Honolulu, 3-8 June 2007, pp. 747-750.

[9] G.-M. Yang, R. Jin, C. Vittoria, V. G. Harris, and N. X. Sun, "Small Ultra-Wideband (UWB) Bandpass Filter with Notched Band," IEEE Microwave and Wireless Components Letters, Vol. 18, No. 3, 2008, pp. 176-178. doi:10.1109/LMWC.2008.916781

[10] W.-S. Chang and C.-Y. Chang, “Analytical Design of Microstrip Short-Circuit Terminated Stepped-Impedance Resonator Dual-Band Filters," IEEE Transactions on Microwave Theory and Techniques, Vol. 59, No. 7, 2011, pp. 1730-1739. doi:10.1109/TMTT.2011.2132140

[11] K. Li, D. Kurita and T. Matsui, "UWB Bandpass Filters with Multi Notched Bands," 36th European Microwave Conference Proceedings, Manchester, 10-15 September 2006, pp. 591- 594.

[12] H. Shaman and J.-S. Hong, "Ultra-Wideband (UWB) Bandpass Filter with Embedded Band Notch Structures," IEEE Microwave and Wireless Components Letter, Vol. 17, No. 3, 2007, pp. 193-195. doi:10.1109/LMWC.2006.890467

[13] M. Mokhtaari and J. Bornemann, "Ultra-Wideband and Notched Wideband Filters with Grounded Vias in Microstrip Technology," Asia-Pacific Microwave Conference Proceedings, Hong Kong, 16-20 December 2008, pp. 1-4.

[14] M. Mokhtaari and J. Bornemann, "Tunable Notch Characteristics in Microstrip Ultra-Wideband Filters," AsiaPacific Microwave Conference Proceedings, Singapore, 7-10 December 2009, pp. 1-4. doi:10.1109/APMC.2009.5384323

[15] S.-Y. Lee and C.-M. Tsai, "New Cross-Coupled Filter Design Using Improved Hairpin Resonators," IEEE Transactions on Microwave Theory and Techniques, Vol. 48, No. 12, 2000, pp. 2482-2490. doi:10.1109/22.899002 